\title{
Estrutura de prática e validade ecológica no processo adaptativo de aprendizagem motora
}

CDD. 20.ed. 152.3

796.346

\author{
Marcela MASSIGLI* \\ Marcelo Eduardo de Souza NUNES* \\ Andrea Michele FREUDENHEIM ${ }^{*}$ \\ Umberto Cesar CORRÊA* \\ *Escola de Educa- \\ ção Física e Esporte, \\ Universidade de São \\ Paulo.
}

\section{Resumo}

0 objetivo desse estudo foi investigar o efeito da estrutura de prática no processo adaptativo de aprendizagem motora em função da validade ecológica da situação experimental. Participaram do estudo 104 crianças distribuídas em oito grupos experimentais (dois níveis de validade ecológica $x$ quatro estruturas de prática). A tarefa consistiu em rebater uma bola de tênis de mesa lançada por um equipamento ou pelo experimentador, com o objetivo de acertar um alvo localizado no lado oposto da mesa. 0 estudo envolveu duas fases: estabilização e adaptação. 0 desempenho foi analisado por meio da soma dos pontos obtidos em blocos de 10 tentativas. Os resultados mostraram que os efeitos das práticas constante, aleatória, constante-aleatória e aleatória-constante no processo adaptativo de aprendizagem motora foram similares em ambos os níveis de validade ecológica; a prática constante foi a estrutura menos efetiva no processo adaptativo de aprendizagem motora em ambas as situações experimentais.

UnITERMos: Aprendizagem motora; Estrutura de prática; Validade ecológica; Tênis de mesa; Processo adaptativo.

\section{Introdução}

No campo da Aprendizagem Motora, a organização da prática tem sido investigada principalmente em três abordagens de pesquisas relacionadas à variabilidade da prática: esquema (SCHMidT, 1975), interferência contextual (BATTIG, 1979) e, mais recentemente, processo adaptativo (TANI, 2005) foco da presente pesquisa.

Nessa abordagem, a aprendizagem de habilidades motoras é vista como um processo adaptativo, ou seja, como um processo em que novas habilidades são formadas a partir da reorganização daquelas já existentes. A aprendizagem envolve duas fases: estabilização e adaptação. A primeira fase diz respeito à estabilização funcional do sistema por meio de "feedback" negativo, ou seja, os movimentos tornamse mais precisos e padronizados. Por exemplo, quando um indivíduo está aprendendo uma rebatida do tênis de mesa, ele apresenta "movimentos grosseiros", diferenciados de tentativa em tentativa e o objetivo da tarefa dificilmente é alcançado. Mas, com a prática, o aprendiz vai adquirindo controle sobre o conjunto de elementos da rebatida (por exemplo, empunhadura, movimento para trás, movimento para frente), integrando-os de forma padronizada de modo que a meta do saque passe a ser alcançada com mais êxito. De acordo com TANI (2005), quando a função se estabiliza pode-se inferir a formação de uma estrutura abstrata no sistema nervoso central como, por exemplo, um programa de ação.

A fase seguinte, denominada de adaptação, é aquela na qual novas habilidades são formadas a partir da reorganização daquelas já adquiridas. De acordo com TANI (2005), a adaptação pode ocorrer de três formas: 1) por meio da própria flexibilidade do sistema, ou seja, via alteração de parâmetros (adaptação paramétrica); 2) por meio da reorganização da estrutura da habilidade (adaptação estrutural); e 3) através da emergência de uma estrutura completamente nova (adaptação auto-organizacional). 
Sob essa perspectiva a organização da prática tem sido investigada em relação às estruturas constante, aleatória, por blocos, constante-aleatória, constante-por blocos e aleatória-constante (BARROS, 2006; CORRÊA, 2001; Corrêa, Barros, Massigli, Gonçalves \& TANI, 2007; CorrÊA, BENDA \& TANI, 2001; CoRrÊA, Gonçalves, Barros \& Massigli, 2006; Massigli, GonÇalVEs, Barros, Souza Junior \& CorrêA, 2004; Paroli, 2004; Pinheiro \& Corrêa, 2007; Tertuliano, Souza Junior, SiLVA Filho \& CorrêA, 2008), sendo que, em sua maioria, os resultados têm apontado para a superioridade da aprendizagem realizada com a prática constante-aleatória em comparação com as demais estruturas de prática.

Entretanto, dois aspectos chamam a atenção nessas pesquisas: o primeiro diz respeito à maioria dos estudos ter sido realizada em situações com baixo nível de validade ecológica. Isso se torna um problema na medida em que permite questionar o poder de generalização dos resultados para situações do mundo real. Já o segundo aspecto, refere-se aos únicos dois estudos realizados com tarefas do mundo real (CORRÊA, BENDA \& TANi, 2001; Tertuliano et al., 2008) não terem possibilitado a inferência de aprendizagem.

Estas constatações levaram à elaboração da seguinte pergunta: os efeitos da estrutura de prática no processo adaptativo de aprendizagem motora seriam dependentes da validade ecológica? De fato, a validade ecológica tem recebido muita atenção em Aprendizagem Motora (Corrêa, Benda \& Ugrinowitsch, 2006; Meira JunIOR, TANI \& MANOEL, 2001), apesar de ela não ter ainda sido investigada como variável independente.

A validade ecológica pode ser entendida como um fenômeno transitório caracterizado por tentativas fundamentadas e sistemáticas para análise de comportamento atual dentro de contextos ambientais específicos, utilizando método de investigação discreto, realista e fidedigno (DAVIDS, 1988) e, ainda, a medida de aproximação da pesquisa ao mundo real (BARReiros, 2008).

DAVIDS (1988) propõe três critérios para a concepção de validade ecológica: 1) realismo: o ambiente experimental deve ser tão realista quanto possível para que características importantes do comportamento permaneçam não afetadas pela imposição das restrições experimentais; 2) união: é o casamento entre o processo de investigação de campo e a necessidade de validade interna; 3) análise eclética: é o uso de uma gama de técnicas de análise para certificar-se de que pelo menos algum aspecto de uma característica particular do comportamento esportivo é analisado em sua versão de mundo real.
Esses critérios permitem pensar na necessidade de um equilíbrio entre a validade externa e a validade interna. A primeira diz respeito à possibilidade de generalização dos resultados de um estudo e a segunda refere-se à dimensão em que os resultados de um estudo podem ser atribuídos aos tratamentos utilizados no mesmo (ThOMAS \& Nelson, 2002). Diante disso, pode-se sugerir que quanto mais a condição de coleta se aproximar do "mundo real", maior será o nível de validade ecológica da situação experimental e, portanto, maior será a validade externa dos resultados. Entretanto, o aumento da validade externa implica em maior dificuldade no controle de suas variáveis e, consequentemente, diminuição da validade interna, e vice-versa. DAVIDS (1988) sugere que os experimentos devem esforçar-se pela validade externa, mas apenas até à medida que eles não sacrifiquem a validade interna.

Considerando as investigaçóes sobre os efeitos da estrutura de prática no processo adaptativo de aprendizagem motora, pergunta-se: quais seriam os efeitos de diferentes estruturas de prática na aprendizagem de habilidades motoras em função da validade ecológica da situação experimental? Seriam os achados das pesquisas de laboratório passíveis de serem replicados em situaçóes com maior validade ecológica? Considerando que a validade ecológica remete-se à aproximação do mundo real, será que o nível de proximidade influenciaria os efeitos da estruturação da prática na aprendizagem motora?

O objetivo desse estudo foi investigar os efeitos de diferentes estruturas de prática no processo adaptativo de aprendizagem motora em função da validade ecológica da situação experimental.

Dada a inexistência de trabalhos que investigaram conjuntamente os efeitos da estruturação da prática e da validade ecológica que pudessem direcionar a elaboração de hipóteses, recorreu-se às explicações existentes sobre os efeitos da estruturação da prática no processo adaptativo de aprendizagem motora, o nosso ponto de partida. Sendo assim, pensou-se que dada à superioridade de variáveis envolvidas na aprendizagem realizada em situações ecologicamente mais válidas, em comparação com situações menos válidas, a estrutura de prática constante seria aquela mais eficaz na promoção do processo adaptativo. Essa hipótese baseou-se na assunção de a prática constante ser caracterizada, em comparação com as demais estruturas, como aquela com menor nível de perturbação e/ou interferência intra e entre tarefas e sem modificação de tentativa a tentativa e, por isso, melhor possibilitar a interação de variáveis ou de componentes no decorrer da prática. 


\section{Método}

\section{Amostra}

A amostra foi formada por 104 crianças destras (52 meninas e 52 meninos), de 10 a 12 anos de idade e sem experiência prévia na tarefa. Os participantes foram alunos da EMEF Fioravante Barletta, escola municipal localizada na cidade de Barueri, Estado de São Paulo, local onde a coleta foi realizada. A participação da criança ocorreu, mediante o termo de consentimento livre e esclarecido, assinado pelo seu responsável. $\mathrm{O}$ presente trabalho foi aprovado pelo Comitê de Ética em Pesquisa da Escola de Educação Física e Esporte da Universidade de São Paulo (sob o protocolo no 2008/32).

\section{Tarefa e equipamento}

A tarefa consistiu em rebater uma bola de tênis de mesa lançada pelo equipamento lançador de bolas ou pelo experimentador, de acordo com a situação experimental, com o objetivo de acertar um alvo localizado na metade oposta da mesa. A raquete era segurada com a mão direita com a empunhadura do tipo caneta. Essa tarefa exigiu do executante "timing" coincidente no momento de contato da raquete com a bola, e precisão para direcionar a bola para o alvo no momento de contato.
A escolha dessa tarefa foi pelo fato de a mesma possuir um alto nível de validade ecológica, sendo imprescindivelmente utilizada em treinos e jogos de tênis de mesa e como atividade de lazer; trata-se de um esporte praticado em todo o mundo (MARINOVIC, IIZUKA \& NAGAOKA, 2006). Além disso, essa tarefa já foi utilizada em pesquisas de aprendizagem motora (MARINOVIC, 2003; MARINOVIC, DANTAS \& Meira Junior, 2000; MarinOVIC \& Freudenheim, 2001; Marinovic, IIZUKa \& FreudenheIm, 2004) e, portanto, possui reconhecimento acadêmico.

Foi utilizado o equipamento lançador de bolas modelo Donic Newgy com controlador de velocidade, frequência de lançamento de bola e oscilador de direção ("table tennis robot"), uma mesa oficial de tênis de mesa com as devidas marcações e rede, duas raquetes de tênis de mesa, 100 bolas oficiais de tênis de mesa, quatro alvos de EVA (quadrantes de 50 x $67,5 \mathrm{~cm}$ ) e um "notebook" no qual foram anotados os dados da coleta. A pontuação 2 foi atribuída para o acerto no alvo, a pontuação 1 para o acerto na mesa e a pontuação zero para as bolas que seriam consideradas erradas numa situação de jogo, ou seja, que não acertaram nem o alvo e nem a mesa (FIGURA 1).

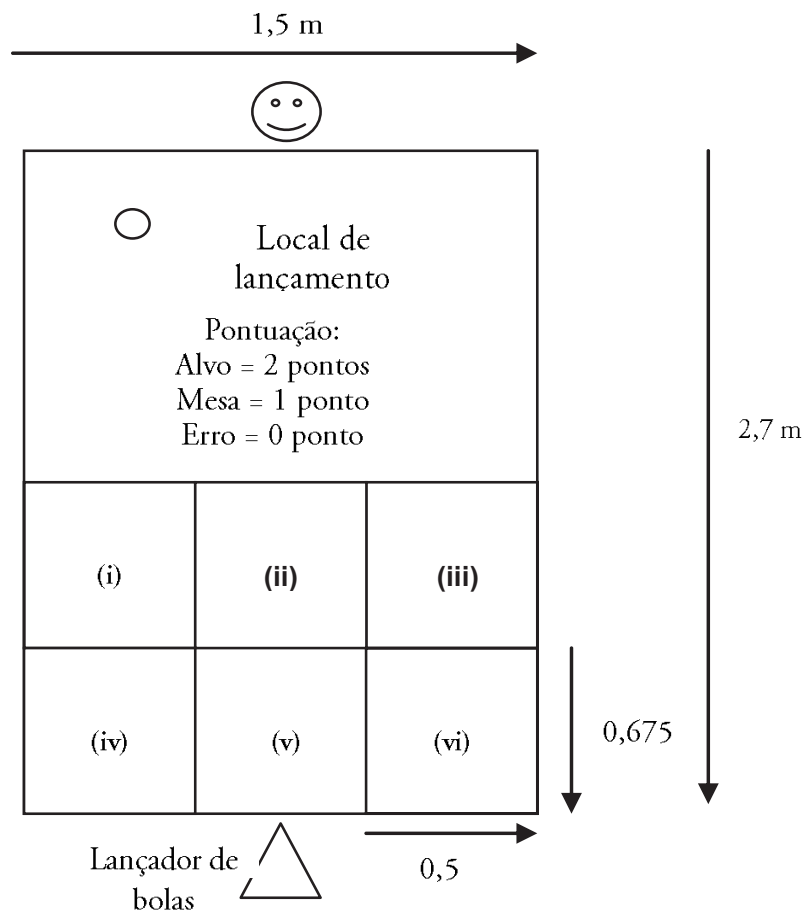

FIGURA 1 - Ilustração do contexto experimental envolvendo as dimensões da mesa (2,7 m x 1,5 m) e dos alvos $(0,675 \mathrm{~m}$ x 0,5 m), os locais em que eram posicionados os lançadores (canhão e experimentador) e os participantes, as pontuações relativas ao desempenho (alvo $=2$; mesa $=1$; erro $=0$ ), elocais para onde os lançamentos eram direcionados. 


\section{Delineamento e procedimentos}

Dadas as definições de validade ecológica, as quais destacam o "grau" ou a "medida" de aproximação da situação experimental com a real, a validade ecológica da situação experimental foi manipulada em dois níveis. Para os sujeitos dos grupos de menor validade ecológica $(\mathrm{n}=12)$, a bolinha foi sempre lançada pelo equipamento lançador de bolas numa única velocidade $(3,2 \mathrm{~m} / \mathrm{s})$, e a coleta foi realizada individualmente, havendo uma pausa de um minuto para descanso a cada 30 tentativas. Já para os grupos de maior validade ecológica $(\mathrm{n}=14)$, a bolinha foi lançada pelo próprio experimentador e a coleta foi realizada com dois sujeitos que se revezavam a cada 30 tentativas. Com relação ao lançamento do experimentador ele foi o tipo caneta (MARINOVIC, IIZUKA \& NaGAOKA, 2006), efetuado com a mão direita. Todos os lançamentos (equipamento lançador e experimentador) eram direcionados para uma mesma área de lançamento (FIGURA 1), e eram feitos na mesma velocidade, considerando-se as diferenças entre ser humano e máquina.

Os participantes foram separados aleatoriamente em quatro grupos de prática para cada nível de validade ecológica: constante (GPC), aleatória (GPA), constante-aleatória (GPCA) e aleatória-constante (GPAC); totalizando oito grupos experimentais. $\mathrm{O}$ experimento foi realizado em duas fases, sendo a primeira, a fase de estabilização, na qual foram realizadas 180 tentativas, de acordo com a situação experimental de cada grupo; e a segunda, a fase de adaptação, que contou com 30 tentativas numa condição igual para todos os sujeitos, porém diferente das condições já praticadas anteriormente. Essa fase ocorreu logo após a fase anterior.

Em ambas as fases a bola foi sempre lançada em direção ao lado direito do sujeito. Sendo que na fase de estabilização, as crianças do GPC executaram todas as tentativas no alvo "vi" (FIGURA 1); já as crianças do GPA executaram todas as tentativas com a variação aleatória de três alvos (ii, iv, vi - FIGURA 1); o GPCA executou a primeira metade das tentativas da mesma forma que o GPC e o restante das tentativas semelhante ao GPA; e, finalmente o GPAC fez o inverso, isto é, executou a primeira metade das tentativas igual ao GPA e o restante da mesma maneira que o GPC. Na fase de adaptação todos os grupos executaram as rebatidas em direção a um novo alvo (i - FIGURA 1).

Primeiramente, o experimentador explicava ao participante qual era o objetivo da tarefa e como ele deveria se posicionar e segurar a raquete. Antes de iniciar o experimento, foram lançadas três bolas pelo equipamento ou pelo experimentador, dependendo da condição experimental, para que o participante entendesse exatamente como seria o procedimento. Feito isso, com o aval do mesmo a coleta era iniciada. Durante a prática aleatória, antes que a bola fosse lançada, o registrador de pontos falava para o sujeito qual alvo ele deveria tentar acertar (ii, iv, vi - FIGURA 1). A cada 10 tentativas era fornecido um "feedback" sumário ao participante acerca do seu padrão de movimento e a cada 30 tentativas havia uma pausa de cerca de um minuto para descanso e para a reposição das bolas. A coleta de cada sujeito durou aproximadamente 30 minutos.

\section{Tratamento dos dados}

A variável dependente foi a pontuação, medida obtida depois da caída da bolinha na mesa e calculada pela somatória dos pontos obtidos pelos sujeitos (precisão) em cada bloco de 10 tentativas, totalizando então, 18 blocos na fase de estabilização e três blocos na fase de adaptação. Foi utilizado também como medida de desempenho o coeficiente de variação (medida de consistência). Considerando a natureza dos dados, o número de participantes por grupo e a proximidade entre média e mediana, optou-se pela utilização de testes paramétricos para analisá-los.

$\mathrm{Na}$ fase de estabilização, foi conduzida para cada grupo uma análise de variância (ANOVA one-way) para verificar o comportamento de cada grupo na sua situação experimental. $\mathrm{Na}$ fase de adaptação foi realizada uma análise de variância a três fatores (ANOVA three-way) (quatro estruturas de prática $\mathrm{x}$ dois níveis de validade ecológica $\mathrm{x}$ quatro blocos de tentativas). Para localizar as diferenças apontadas pela ANOVA foi aplicado o teste de Tukey HSD. Na comparação intra-grupo utilizou-se os dados do último bloco de tentativas da fase de estabilização e os três blocos da fase de adaptação. Para todas as análises foi utilizado o "software" Statistica. 


\section{Resultados}

\section{Precisão}

Conforme se pode observar na FIGURA 2, todos os grupos de menor validade ecológica melhoraram consideravelmente o seu desempenho ao longo da fase de estabilização (1 a 18). Esses resultados foram confirmados pelas análises estatísticas (ANOVAs one-way): GPC $[\mathrm{F}(17 ; 187)=4,206, \mathrm{p}<0,001]$; GPA $[\mathrm{F}(17 ; 187)=3,688, \mathrm{p}<0,001]$; GPCA [F(17; $187)=2,873, \mathrm{p}<0,001]$; e GPAC $[\mathrm{F}(17 ; 187)=$ 7,907, $\mathrm{p}<0,001)$.

As análises estatísticas (ANOVAs one-way) também confirmaram que os grupos de maior validade ecológica melhoraram o desempenho ao longo da fase de estabilização (1 a 18): $\mathrm{GPC}[\mathrm{F}(17 ; 221)=5,257, \mathrm{p}<$ 0,001]; GPA [F(17; 221) = 4,314, p < 0,001]; GPCA $[\mathrm{F}(17 ; 221)=3,605, \mathrm{p}<0,001]$; e GPAC $[\mathrm{F}(17 ; 221)$ $=4,891, \mathrm{p}<0,001)]$. Em ambos os casos, o "post hoc" (TukeyHSD) apontou que os desempenhos nos blocos de tentativas iniciais foram piores do que aqueles dos últimos blocos de tentativas $(\mathrm{p}<0,05)$.

$\mathrm{Na}$ fase de adaptação, a análise de variância a três fatores apontou diferenças para o fator nível de validade ecológica $[\mathrm{F}(1 ; 96)=30,934, \mathrm{p}<0,001]$, para o fator blocos de tentativas $[\mathrm{F}(3 ; 288)=14,533, \mathrm{p}<$ $0,001]$, e para a interação entre estrutura de prática e blocos de tentativas $[\mathrm{F}(9 ; 288)=3,201, \mathrm{p}<0,001]$.
No que concerne ao fator nível de validade ecológica, o "post hoc" mostrou que os grupos de maior validade ecológica tiveram melhores desempenhos do que os grupos de menor validade ecológica $(\mathrm{p}=$ 0,000). Para o fator blocos de tentativas, o "post hoc" apontou que no último bloco de tentativas da fase de estabilização os grupos apresentaram um desempenho melhor do que no $1^{\circ}$ bloco da fase de adaptação $(\mathrm{p}=$ 0,000 ), e que este último, por sua vez, apresentou um desempenho inferior aos $2^{\circ}$ e $3^{\circ}$ blocos de tentativas da fase de adaptação $(p=0,000)$. Assim pode-se inferir que, a modificação da tarefa (perturbação) acarretou uma piora do desempenho no $1^{\circ}$ bloco da adaptação, porém, em seguida, o desempenho melhorou significativamente nos demais blocos de tentativas. E, para a interação entre estrutura de prática e blocos de tentativas, o "post hoc" mostrou que a citada perturbação foi mais evidente para o grupo de prática constante (GPC), pois a mudança na tarefa fez com que o desempenho piorasse, mas o mesmo foi retomado em seguida, ou seja, os sujeitos apresentaram um desempenho significativamente melhor no último bloco de tentativas da fase de estabilização quando comparados ao $1^{\circ}$ bloco da fase de adaptação ( $\mathrm{p}<0,001)$, e este último, por sua vez, apresentou um desempenho inferior ao $3^{\circ}$ (último) bloco de tentativas da fase de adaptação $(\mathrm{p}<0,001)$.

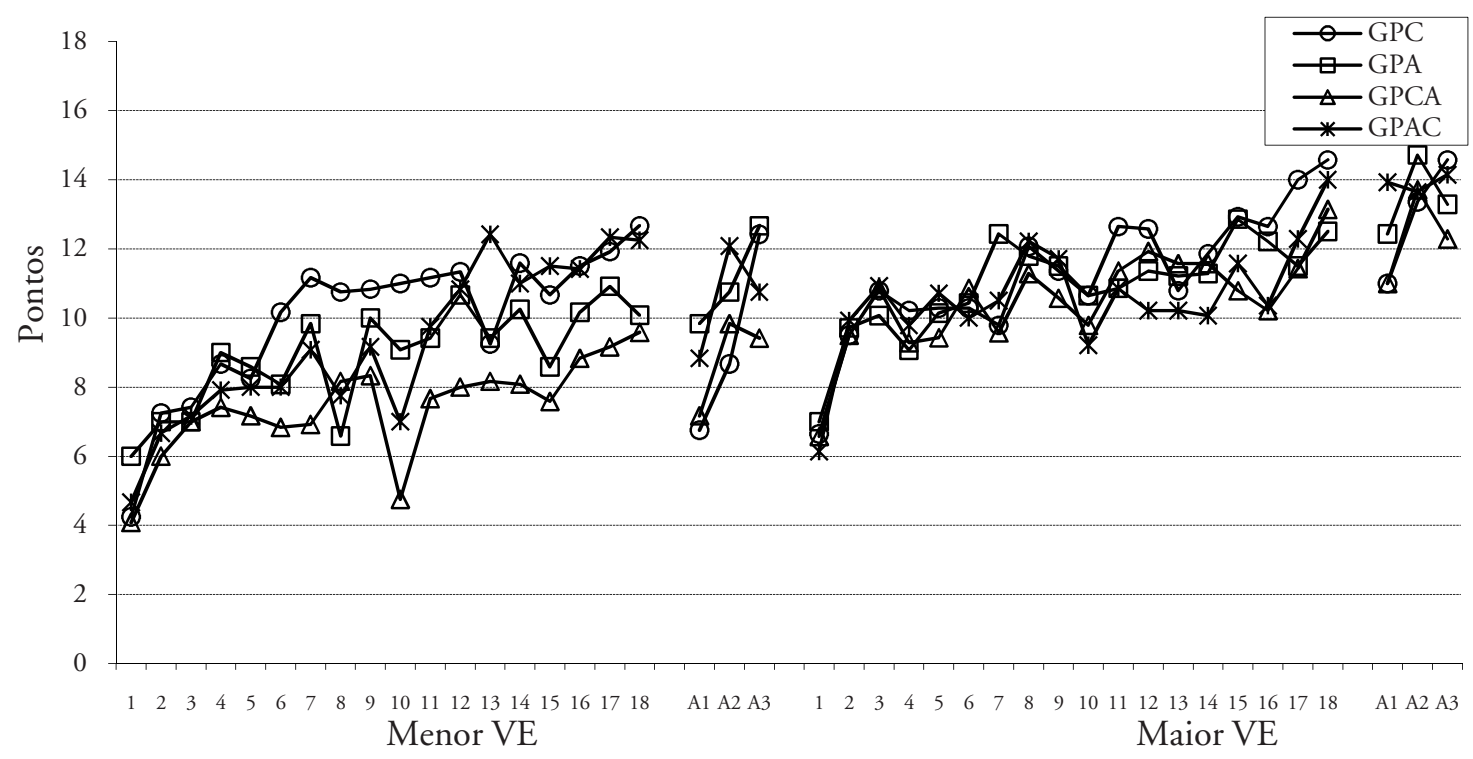

Blocos de tentativas

FIGURA 2 - Curvas de desempenho (soma depontos) dos quatro grupos experimentais (GPC, GPA, GPCA, GPAC) de menor validade ecológica (Menor VE) e de maior validade ecológica (Maior VE), por blocos de 10 tentativas, nas fases de estabilização (1a 18) e adaptação (A1 a A3). 


\section{Consistência}

Conforme se pode observar na FIGURA 3, todos os oito grupos experimentais se tornaram mais consistentes nas respostas ao longo da estabilização. Esses resultados foram confirmados pelas análises de variância (ANOVAs one-way), os quais detectaram diferenças significativas entre os blocos de tentativas para todos os grupos de menor validade ecológica: GPC $[\mathrm{F}(17 ; 170)=2,999, \mathrm{p}<0,001]$; GPA [F(17; $170)=3,243, \mathrm{p}<0,001]$; GPCA $[\mathrm{F}(17 ; 119)=1,984$, $\mathrm{p}=0,017]$; e GPAC $[\mathrm{F}(17 ; 187)=4,419, \mathrm{p}<0,001)]$.
Para os quatro grupos de maior validade ecológica as análises de variância (ANOVAs one-way) também encontraram diferenças significantes entre os blocos de tentativas: $\mathrm{GPC}[\mathrm{F}(17 ; 204)=2,446, \mathrm{p}=0,001]$; GPA $[\mathrm{F}(17 ; 221)=4,161, \mathrm{p}<0,001]$; GPCA $[\mathrm{F}(17$; $221)=2,817, \mathrm{p}<0,001]$; e GPAC $[\mathrm{F}(17 ; 221)=$ 4,666, $\mathrm{p}<0,001)]$.

Em ambos os casos (maior e menor nível de validade ecológica), o "post hoc" (TukeyHSD) apontou que os desempenhos nos blocos de tentativas iniciais foram piores do que aqueles dos últimos blocos de tentativas $(\mathrm{p}<0,05)$.

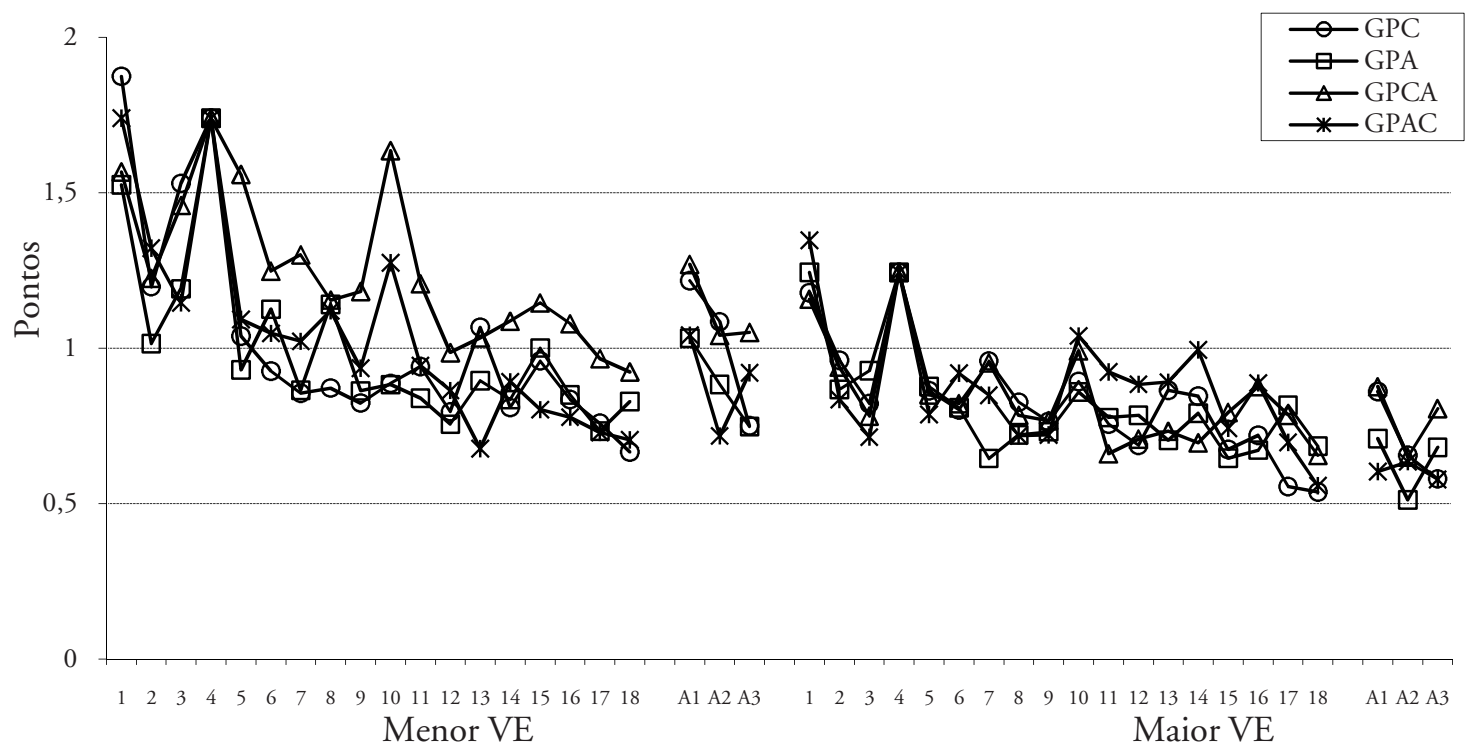

Blocos de tentativas

FIGURA 3 - Coeficiente devariação dos quatro grupos experimentais (GPC, GPA, GPCA, GPAC) demenor validade ecológica (Menor VE) e de maior validade ecológica (Maior VE), por blocos de 10 tentativas na fase de estabilização (1 a 18) e adaptação (A1 a A3).

$\mathrm{Na}$ fase de adaptação a análise de variância a três fatores apontou diferenças apenas para o fator nível de validade ecológica $[\mathrm{F}(1 ; 94)=21,599, \mathrm{p}<0,001] \mathrm{e}$ para o fator blocos de tentativas $[\mathrm{F}(3 ; 282)=11,549$, $\mathrm{p}<0,001]$. Não foram detectadas diferenças significativas para o fator estrutura de prática $[\mathrm{F}(3 ; 94)=$ 2,441, $\mathrm{p}=0,069$ ]; para a interação entre estrutura de prática e nível de validade ecológica $[\mathrm{F}(3 ; 94)=$ $0,230, p=0,875]$; para a interação entre estrutura de prática e blocos de tentativas $[\mathrm{F}(9 ; 282)=1,834$, $\mathrm{p}=0,062]$; para a interação entre nível de validade ecológica e blocos de tentativas $[\mathrm{F}(3 ; 282)=2,287$, $\mathrm{p}$ $=0,079]$; e por fim, para a interação entre os três fatores, estrutura de prática, nível de validade ecológica e blocos de tentativas $[F(9 ; 282)=0,818, p=0,600]$.

Para o fator nível de validade ecológica o "post hoc" apontou diferenças significativas entre os dois níveis de validade ecológica $(\mathrm{p}<0,001)$, indicando que as respostas dos grupos de maior validade ecológica foram mais consistentes do que as dos grupos de menor validade ecológica. Para o fator blocos de tentativas o "post hoc" apontou que no último bloco de tentativas da fase de estabilização os sujeitos apresentaram um comportamento com menor variabilidade do que no $1^{\circ}$ bloco da fase de adaptação $(\mathrm{p}=0,000)$, e que este último, por sua vez, apresentou um comportamento com maior variabilidade do que os blocos duas e três de tentativas $\mathrm{da}$ fase de adaptação $(\mathrm{p}<0,001)$. Assim pode-se inferir que a modificação da tarefa (perturbação) acarretou no aumento da variabilidade do desempenho no $1^{\circ}$ bloco da adaptação, porém, em seguida, essa variabilidade diminuiu significativamente nos dois últimos blocos de tentativas restantes da fase de adaptação. 


\section{Discussão}

Uma preocupação presente durante a realização desse estudo foi a de garantir que houvesse aprendizagem, visto que, nos outros dois estudos (CORRÊA, BENDA \& TANI, 2001; TERTULIANO et al., 2008) sobre estrutura de prática e processo adaptativo em que a questão da validade ecológica foi contemplada, não foi possível inferir a ocorrência da mesma. Especificamente, nesses estudos anteriores nenhum dos grupos alcançou a estabilização funcional, comprometendo, assim, qualquer especulação sobre o processo adaptativo. Dessa forma, a primeira questão a ser discutida nesse trabalho é se houve o processo de estabilização, visto que sem ele não faria sentido analisar a fase de adaptação.

Com base na análise dos resultados da fase de estabilização, tanto no que diz respeito à precisão quanto à consistência, foi possível verificar uma melhora significativa do desempenho de todos os grupos experimentais. Essas mudanças ocorridas ao longo das tentativas permitem a conclusão de que a estabilização funcional de fato ocorreu no decorrer da fase de estabilização. Diante disso, os olhares são direcionados para o objetivo da pesquisa: investigar os efeitos das práticas constante, aleatória, constante-aleatória e aleatória-constante no processo adaptativo de aprendizagem motora em função da validade ecológica da situação experimental.

Esperava-se que a estrutura constante seria aquela mais eficaz no processo adaptativo de aprendizagem motora. A lógica de raciocínio utilizada para construção dessa hipótese foi a seguinte: uma vez que o aumento em validade ecológica envolve o aumento da quantidade de variáveis, haveria a necessidade de uma estrutura de prática que possibilitasse ao aprendiz lidar com as mesmas. E, de acordo com a literatura (BARros, 2006; CORRÊA, 2001, 2007; CorrêA et al., 2006, 2007; MAssigli et al., 2004; PAROLI, 2004) a prática constante seria aquela que possibilitaria mais eficientemente a formação da estrutura da habilidade ou, a formação de um padrão de interação entre os componentes do sistema (variáveis envolvidas no processo de aprendizagem).

Entretanto, essa hipótese não foi confirmada. Considerando o objetivo e as expectativas em relação aos resultados, a discussão que segue foi organizada de modo a abordá-los (resultados) em dois momentos: no primeiro, os resultados são discutidos considerando-se os dois níveis de validade ecológica manipulados. E, no segundo, os resultados são discutidos em relação à literatura, ou seja, considerando-se os resultados das pesquisas realizadas em situação de laboratório.
Um primeiro aspecto a destacar é que, por se tratar de um estudo em que a validade ecológica é uma das variáveis independentes e, como foi colocado anteriormente, em virtude de ela remeter-se ao grau ou a medida de aproximação da situação experimental com "a real”, ela foi manipulada em dois níveis. Tentou-se contemplar ao máximo os critérios propostos por DAVIDS (1988), principalmente na situação de maior validade ecológica. Tentou-se deixar o ambiente experimental o mais realista possível, sendo que muitas características importantes do comportamento, tais como percepção, tomada de decisão e organização efetora foram minimamente afetadas. Ao mesmo tempo, buscou-se o equilíbrio entre validade interna e externa, ou seja, a validade ecológica e, consequentemente, a validade externa foram priorizadas ao máximo, uma vez que os aprendizes rebateram uma bola lançada (sacada) por uma pessoa (jogador) que estava no lado oposto, e cujo objetivo era acertar a quadra adversária, mas sem que para isso a validade interna fosse totalmente sacrificada. Nesse caso, como pôde ser observado no método, o intervalo entre blocos de tentativas, a área de lançamento, a distribuição dos participantes nos grupos, os tipos de lançamento e de rebatida, o posicionamento do participante, dentre outros aspectos, foram controlados experimentalmente.

Entretanto, os testes estatísticos não apresentaram diferenças significativas na interação dos fatores estrutura de prática e nível de validade ecológica, o que leva a conclusão de que os efeitos das práticas constante, aleatória, constante-aleatória e aleatóriaconstante, no processo adaptativo, foram similares, considerando-se a aprendizagem em ambos os níveis de validade ecológica manipulados.

Em contrapartida, para a interação entre os fatores estrutura de prática e blocos de tentativas, foram encontradas diferenças estatísticas, apenas para os grupos de prática constante. Com a mudança da tarefa (perturbação), houve uma nítida queda do desempenho do último bloco da fase de estabilização para o primeiro bloco da fase de adaptação, porém, o mesmo foi retomado nos dois últimos blocos da adaptação. Esse comportamento sugere que os grupos de prática constante foram os únicos que significativamente se abalaram com a perturbação inserida no primeiro bloco de tentativas da fase de adaptação. A pergunta que surge nesse caso é: por que a prática constante conduziria à pior adaptação?

Uma possível resposta seria que a ênfase dada a um único padrão ou situação poderia levar a uma perda de flexibilidade na estrutura da habilidade que, como 
consequência, dificultaria a sua adaptação. Dessa forma, pode-se sugerir que a prática constante propiciou a formação de uma estrutura mais rígida que, por sua vez, dificultou a adaptação imediata à nova situação.

Outro aspecto interessante para se destacar é que para o fator nível de validade ecológica foram localizadas diferenças significativas. Em termos de precisão, os grupos de maior nível de validade ecológica foram melhores do que os grupos de menor nível de validade ecológica. Em princípio, esse foi um fato que surpreendeu, pois o lançamento da bolinha pelo equipamento, por se tratar de uma máquina, era visivelmente mais preciso e consistente do que o lançamento feito pelo experimentador.

Uma explicação para isso seria que, na situação de maior validade ecológica, os sujeitos, além de observarem o colega (modelo não habilidoso) executando a tarefa durante o revezamento, podiam também observar o movimento de rebatida do experimentador (modelo habilidoso) ao lançar a bolinha a cada tentativa. Com base nisso, eles procuravam prever o momento exato em que a bola seria lançada e, assim, antecipar o seu próprio movimento. Já na situação de menor validade ecológica, a coleta era feita individualmente e o equipamento lançador de bolas não fornecia ao observador a informação do momento exato de lançamento da bolinha, e muito menos do padrão de movimento da rebatida. A observação de um modelo permite que o aprendiz elabore referências, símbolos ou representaçóes na memória acerca da habilidade motora a ser aprendida. Esses resultados e interpretaçóes permitem corroborar as afirmações de Pellegrini e Tonello (1997) de que o papel do modelo é facilmente observado tanto em situações de ensino, como em situações do cotidiano.

Outro aspecto dos resultados do presente estudo que merece atenção refere-se aos mesmos terem sido diferentes aqueles observados em pesquisas em situações de laboratório. Com base nisso, não se pode afirmar que os resultados dos estudos anteriores realizados em situações de laboratório são generalizáveis para situações de maior validade ecológica, visto que os grupos de prática constantealeatória não foram superiores aos outros.

\section{Conclusão}

Não houve interação entre estrutura de prática e validade ecológica, portanto, os efeitos das práticas constante, aleatória, constante-aleatória e aleatóriaconstante no processo adaptativo de aprendizagem
Porém, um aspecto importante a se considerar, e que talvez possa ser caracterizado como limitação do estudo, é que a maioria desses estudos utilizou não apenas medidas de desempenho relacionado à meta da tarefa, mas também de padrão de movimento (BArRos, 2006; CorrêA, 2001; CORRÊA et al., 2006, 2007; MAssigli et al., 2004). Quando desconsideradas as análises do padrão de movimento, não foram encontradas diferenças significativas nas medidas de desempenho que possibilitassem a afirmação da superioridade da prática constante-aleatória.

Pode ser que somente as medidas de desempenho desse estudo não tenham sido suficientes para enxergar diferenças mais sutis entre as estruturas de prática, porém, o fato a única estrutura que não tem variabilidade ter possibilitado a pior adaptação em relação às outras estruturas de prática, permite pensar que em situações mais próximas do "mundo real", a variabilidade da prática deve estar presente em algum momento do processo de aprendizagem. Seja, antes ou depois da prática constante, ou ainda seja ela exclusivamente aleatória. Observa-se também que essa variabilidade que a prática aleatória proporciona é a que mais se aproxima não somente de situações "reais" de jogo, mas também de inúmeras situações do cotidiano, já que ambos são abertos e dinâmicos. Os benefícios da variabilidade na prática nos contextos citados podem ser explicados por algumas hipóteses desenvolvidas no âmbito do processo adaptativo de aprendizagem motora. Por exemplo, Barros (2006) propôs que, numa situação na qual os aprendizes não podiam acessar visualmente o ambiente de aprendizagem, a prática variada aleatória possibilitou que os aprendizes explorassem e reconhecessem o espaço, e que isso foi fundamental para melhorar o desempenho em direção do alcance da meta da tarefa de tentativa a tentativa. Já, CorrêA, Benda e Tani (2001), CorrêA et al. (2006, 2007) e CorrêA, Massigli, Barros, Gonçalves, Oliveira e Tani (2010) propuseram a prática variada aleatória como um meio para a diversificação da habilidade motora,ou seja, aumento de recursos que possibilita a adaptação.

motora foram similares em ambos os níveis de validade ecológica manipulados, mas diferentes daqueles verificados em situaçôes de laboratório; a prática constante foi a estrutura menos efetiva no 
processo adaptativo de aprendizagem motora em ambas as situações experimentais.

Os resultados do presente estudo permitem sugerir que os efeitos das estruturas constante, aleatória, constante-aleatória e aleatória constante são dependentes do nível de validade ecológica da situação experimental, considerando-se níveis inferiores como aqueles relativos a situações laboratoriais. Dessa forma, pensa-se na necessidade de utilizar cada vez mais situaçôes experimentais com altos níveis de validade ecológica nas investigaçôes em aprendizagem motora a fim de melhor entender e explicar esse fenômeno. De acordo com TANI, Dantas e MANOEL (2005), quanto mais controladas as variáveis intervenientes, mais artificiais tornam-se as condiçōes de experimentação no que se refere a ambiente, tarefa e instrução. Evidentemente, conhecimentos dessa natureza, com a validade ecológica comprometida, são difíceis de serem aplicados na solução de problemas do "mundo real", visto que aquelas variáveis que foram controladas na pesquisa encontram-se agora "livres".

\begin{abstract}
Practice schedule and ecological validity in the adaptive process of motor learning

The aim of this study was to investigate the effect of different practice schedules in the adaptive process of motor learning in function of the ecological validity of the experimental situation. Participants were 104 children distributed in eight experimental groups (2 levels of ecological validity $x 4$ practice schedules). The task was to hit a table tennis ball thrown by equipment or experimenter, aiming to hit a target located on the opposite side of the table. The study was carried out in two phases: stabilization and adaptation. Performance was analyzed through the sum of the points achieved in ten trial blocks. Results showed that the effects of constant, random, constant-random and random-constant practice on the adaptive process of motor learning were similar in both levels of ecological validity; constant practice was the less effective schedule on the adaptive process of motor learning in both experimental situations.
\end{abstract}

UnITERMS: Motor learning; Practice schedule; Ecological validity; Table tennis; Adaptive process.

\title{
Referências
}

BARREIROS J. Metodologia da investigação científica. Lisboa: Faculdade de Motricidade Humana da Universidade Técnica de Lisboa, 2008. Disponível em: <http://home.fmh.utl.pt/ -jbarreiros/MIC-pdf>. Acesso em: 27 abr. 2008.

BARROS, J.A.C. Estrutura de prática e processo adaptativo em aprendizagem motora. 2006. Dissertação (Mestrado) - Escola de Educação Física e Esporte, Universidade de São Paulo, São Paulo, 2006.

BATTIG, W.F. The flexibility of human memory. In: CERMAK, L.S.; CRAIK, F.I.M. (Eds.). Levels of processing in human memory. Hillsdale: Lawrence Erbaum, 1979. p.23-44.

CORREAA, U.C. Estrutura da prática e processo adaptativo na aquisição de habilidades motoras. 2001. Tese (Doutorado) - Escola de Educação Física e Esporte da Universidade de São Paulo, São Paulo, 2001.

Prática constante-variada e a aquisição de habilidades motoras. 2007. Tese (Livre Docência)- Escola de Educação Física e Esporte, Universidade de São Paulo, São Paulo, 2007.

CORRÊA, U.C.; BARROS, J.A.C.; MASSIGLI, M.; GONÇALVES, L.A.; TANI, G. A prática constante-aleatória e o processo adaptativo de aprendizagem motora: efeitos da quantidade de prática constante. Revista Brasileira de Educação Física e Esporte, São Paulo, v.21, n.4, p.301-14, 2007.

CORREAA, U.C.; BENDA, R.N.; TANI, G. Estrutura de prática e processo adaptativo na aquisição do arremesso de dardo de salão. Revista Brasileira de Ciências do Esporte, Campinas, v.22, n.2, p.69-83, 2001.

CORREAA, U.C.; BENDA, R.N.; UGRINOWITSCH, H. Processo ensino-aprendizagem no ensino do desporto. In: TANI, G.; BENTO, J.O.; PETERSEN, R.D. Pedagogia do desporto. Rio de Janeiro: Guanabara Koogan, 2006. p.241-50. CORRÊA, U.C.; GONÇALVES, L.A.; BARROS, J.A.C.; MASSIGLI, M. Prática constante-aleatória e aprendizagem motora: efeitos da quantidade de prática constante e da manipulação de exigências motoras da tarefa. Brazilian Journal of Motor Behavior, Rio Claro, v.1, n.1, p.41-52, 2006. 
CORREAA, U.C.; MASSIGLI, M.; BARROS, J.A.C; GONÇALVES, L.A.; OLIVEIRA, J.A.; TANI, G. Constant-random practice and the adaptive process in motor learning with varying amounts of constant practice. Perceptual and Motor Skills, Missoula, v.110, n.2, p.442-42, 2010.

DAVIDS, K. Ecological validity in understanding sport performance: some problems of definition. Quest, Champaign, v.40, p.126-36, 1988.

MARINOVIC, W. Efeito da modificação da tarefa no movimento de rebatida de forehand de mesa-tenistas habilidosos. 2003. Dissertação (Mestrado) - Escola de Educação Física e Esporte, Universidade de São Paulo, São Paulo, 2003. MARINOVIC, W.; DANTAS, L.E.P.B.T.; MEIRA JUNIOR, C.M. Conhecimento e desempenho em mesa-tenistas: um estudo exploratório. Boletim do Laboratório de Comportamento Motor [da EEFE-USP], São Paulo, v.7 p.2-6, 2000. MARINOVIC, W.; FREUDENHEIM, A. M. Prática variada: a melhor opção para a aquisição de uma habilidade motora? Revista Paulista de Educação Física, São Paulo, v.15, p.103-10, 2001.

MARINOVIC, W.; IIZUKA, C.A.; FREUDENHEIM, A.M. Control of striking velocity by table tennis playes. Perceptual and Motor Skills, Missoula, v.99, p.1027-34, 2004.

MARINOVIC, W.; IIZUKA, C.A.; NAGAOKA, K.T. (Orgs.). Tênis de mesa. São Paulo: Phorte, 2006.

MASSIGLI, M.; GONÇALVES, L.A.; BARROS, J.A.C.; SOUZA JUNIOR, O.P.; CORRÊA, U.C. O efeito de diferentes quantidades de prática constante-aleatória no processo adaptativo na aquisição de habilidades motoras: manipulação de exigências perceptivas da tarefa. In: CONGRESSO INTERNACIONAL DE EDUCAÇÃO FÍSICA, 19.; CONGRESSO DE FISIOTERAPIA DO MERCOSUL; CONGRESSO CINETÍfICO DA FIEP, Foz do Iguaçu. Anais... Foz do Iguaçu: FIEP, 2004. p.131.

MEIRA JUNIOR, C.M.; TANI, G; MANOEL, E.J. A estrutura da prática variada em situaçóes reais de ensino-aprendizagem. Revista Brasileira de Ciência e Movimento, Brasília, v.9, n.4, p55-63, 2001.

PAROLI, R. Efeito da estrutura de prática na aquisição de uma habilidade motora. 2004. Dissertação (Mestrado) Escola de Educação Física e Esporte, Universidade de São Paulo, São Paulo, 2004.

PELLEGRINI, A.M.; TONELLO, M.G.M. A. informação na aprendizagem motora: o modelo em destaque. In: PELEGRINI, A.M. (Org.). Coletânea de estudos: comportamento motor I. São Paulo: Movimento, 1997. p.119-39.

PINHEIRO, J.P.; CORRÊA, U.C. Estrutura de prática na aquisição de uma tarefa de timing coincidente com desaceleração do estímulo visual. Revista Portuguesa de Ciências do Desporto, Porto, v.7, n.3, p.336-46, 2007.

SCHMIDT, R.A. A schema theory of discrete motor skill learning. Psychological Review, Princeton, v.82, n.4, p.225-60, 1975. TANI G. Processo adaptativo: uma concepção de aprendizagem motora além da estabilização. In: TANI, G. (Ed.). Comportamento motor: desenvolvimento e aprendizagem. Rio de Janeiro: Guanabara Koogan, 2005. p.60-70.

TANI, G.; DANTAS, L.E.P.B.T.; MANOEL, E.J. Ensino-aprendizagem de habilidades motoras: um campo de pesquisa de síntese e integração de conhecimentos. In: TANI, G. (Ed.). Comportamento motor: desenvolvimento e aprendizagem. Rio de Janeiro: Guanabara Koogan, 2005. p.106-116.

TERTULIANO, I.W.; SOUZA JÚNIOR, O.P.; SILVA FILHO; CORRÊA, U.C. Estrutura de prática e freqüência de feedback extrínseco na aprendizagem de habilidades motoras. Revista Brasileira de Educação Física e Esporte, São Paulo, v.22, p.103-18, 2008

THOMAS, J.R.; NELSON, J.K. Métodos de pesquisa em atividade física. 3. ed. Porto Alegre: Artmed, 2002.

ENDEREÇO

Umberto Cesar Corrêa Escola de Educação Física e Esporte - USP Av. Prof. Mello Moraes, 65

05508-030 - São Paulo - SP - BRASIL e-mail: umbertoc@usp.br
Recebido para publicação: 22/ 10/2009

Revisado: 07/06/2010

Aceito: 06/08/2010 